\title{
Editorial
}

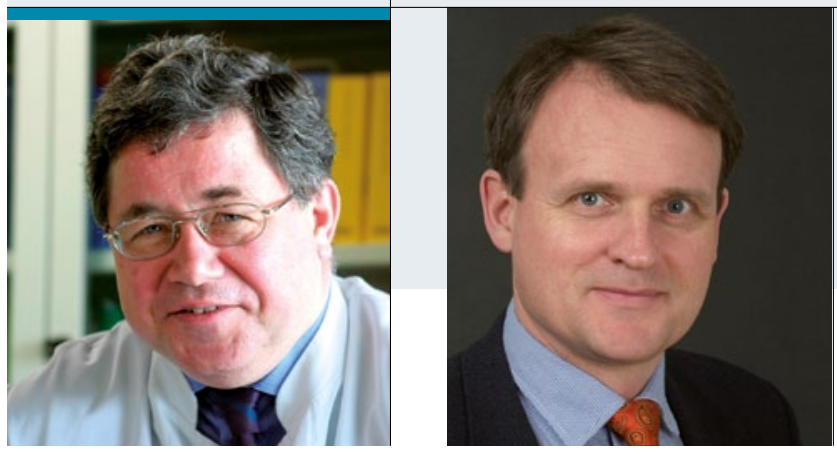

„In einigen Teilbereichen der Allergologie

bestehen schon heute Möglichkeiten einer personalisierten Medizin."

Prof. Dr. Hans F. Merk, Hautklinik der Medizinischen Fakultät, Universitätsklinikum der RWTH Aachen Prof. Dr. Thilo Jakob, Allergieabteilung \& Forschergruppe Allergologie, Universitäts-Hautklinik Freiburg

\section{Sequenzieren, interpretieren, personalisieren}

Z unehmend häufiger lesen wir von „personalisierter Medizin“. Was hat es damit auf sich und inwieweit ist unser Fachgebiet davon betroffen? Lassen Sie uns an einem Beispiel die Bedeutung dieser neuen Entwicklung betrachten: Die Erkenntnis, dass Bradykinin die wesentliche Mediatorsubstanz zur Auslösung eines hereditären oder ACEinduzierten Angioödems ist, hat nicht nur zu einer modifizierten Einteilung der Angioödeme nach pathophysiologischen Kriterien geführt, sondern auch neue therapeutische Optionen eröffnet. Mehr darüber ist in der neuen Leitlinie zum hereditären Angioödem auf S. 109 ff. nachzulesen.

Die Entwicklung derartiger neuer Präparate zur Behandlung einer vergleichsweise kleinen Patientengruppe ist wesentlich leichter oder überhaupt erst möglich geworden durch besondere, vereinfachte Regularien zur Zulassung von Medikamenten bei sogenannten „orphan diseases“. Erschwert wird dagegen die Etablierung neuer Therapien mit gezielt wirkenden Präparaten gegenwärtig besonders dadurch, dass ihre gezielte Wechselwirkung mit humanen Rezeptoren oder Mediatoren dazu führt, dass zu Beginn klinisch-pharmakologischer Studien nur unzuverlässige präklinische Daten vorliegen. Diese Situation zwingt dazu, solche Medikamente nur bei Patienten einzusetzen, die auch sicher von diesen Arzneimitteln profitieren. Eine Entwicklung, die mit einer immer kostengünstiger werdenden Sequenzierung des gesamten Genoms zusammentrifft - Institute in den USA bieten entsprechendes bereits für weniger als 1.000 US-\$ an. Das Problem wird die anschließend notwendige Interpretation der Daten sein: „It may be the $\$ 1.000$ genome, but it will be the $\$ 100.000$ interpretation", heißt es in einer aktuellen Studie [Weiss ST. J Allergy Clin Immunol 2012; 129: 327-34].

Ein von Georg Stingl und Martin Röcken organisiertes Leopoldina-Symposium im Januar $2012 \mathrm{kam}$ $\mathrm{zu}$ dem Ergebnis, dass in einigen Teilbereichen der Allergologie schon heute Möglichkeiten einer ,per- sonalisierten Medizin" bestehen. So ist unter anderem der genetische Hintergrund individuell unterschiedlicher Wirksamkeiten von $\beta$-Rezeptor-Agonisten, Glukokortikoiden und Leukotrienantagonisten bei Asthma bronchiale bekannt. Patienten mit Risiken für schwere Hautreaktionen auf einzelne Medikamente wie z. B. toxische epidermale Nekrolyse lassen sich durch HLA-Polymorphismen identifizieren. Für das Carbamazepin-induzierte SJS/TEN sind bei Chinesen Assoziationen mit HLA-B-1502, bei Japanern mit HLA-B-1511 und bei Europäern mit HLA-A-3101 beschrieben. Gerade dieses letzte Beispiel zeigt die Komplexität der Interpretation, da Medikament, Art der Reaktion und ethnischer Hintergrund im Einzelfall zu unterschiedlichen Assoziationen führen. Nur die Verfügbarkeit dieser Daten - zusammen mit Programmen zur Interpretation und Entscheidungshilfe - sowie ein entsprechender Zugang des behandelnden Arztes dazu wird eine Entwicklung der „personalisierten Medizin“ möglich machen und wahrscheinlich eine wesentliche Herausforderung in den nächsten zehn Jahren darstellen.

Auch andere Veränderungen stellen Herausforderungen für die Allergologie dar. Der Klimawandel z. B. kann sich auf Art und Eigenschaften der Allergene auswirken. Um so wichtiger ist es, dass Allergologen in ihrem Umfeld darauf achten, dass durch fehlende Berücksichtigung allergologischer Fragestellungen - beispielswseise bei Bepflanzungsmaßnahmen - die Situation nicht noch weiter erschwert wird. Die Arbeit von Bergmann et al. (S. 103) gibt dazu wichtige Anregungen.

Schließlich werden in dem Beitrag von Ring et al. (S. 96) Inhalte und Vorraussetzungen einer Anaphylaxieschulung dargestellt.

Freude und Erkenntnissgewinn bei der Lektüre der aktuellen Ausgabe wünschen Ihnen

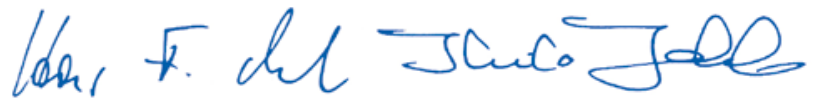

